\title{
STUDY AND DEVELOPMENT OF NEUTRON DETECTORS USING DOPED CSI CRYSTALS
}

\author{
Tufic Madi Filho, ${ }^{1,2^{*}}$, Maria da Conceição Costa Pereira', \\ José Roberto Berretta', Lucas Faustino Tomaz', Miriam Nieri Madi'
}

\author{
${ }^{1}$ Instituto de Pesquisas Energéticas e Nucleares, São Paulo, SP \\ ${ }^{2}$ Universidade Paulista - UNIP-SP, Software Engineering Research Group, Paulista University, São Paulo, Brazil
}

\begin{abstract}
The development of new radiation detectors using scintillation crystals, which increase response speed, dose and energy accuracy and, at the same time, the feasibility of simplifying and reducing costs in the production process are always necessary. In the CTR-IPEN laboratory, pure and doped CsI crystals were grown using the Bridgman technique. This work shows the obtained results using a doped CsI scintillator with the converters: $\mathrm{Br}, \mathrm{Pb}$, Tl, Li as alpha, beta, gamma and neutron detectors.
\end{abstract}

Keywords: Crystals, dopants: $\mathrm{Br}, \mathrm{Pb}, \mathrm{Tl}, \mathrm{Li}$, radiation: alpha, beta, gamma and neutrons, Scintillation detectors

\section{INTRODUCTION}

In the development of nuclear radiation detectors, one must take into consideration the process of interaction of radiation under study with matter. In the case of neutron detectors, it must be considered that the detection of neutrons is not trivial in view of the lack of charges of these particles and the peculiarity of their reaction type. Another difficulty in the detection of neutrons consists of the discrimination of the electronic impulses generated by the neutrons of those generated by other radiations, almost always present in the measurement field. The development of new radiation detectors using scintillation crystals, which increase response speed, dose and energy accuracy and, at the same time, the feasibility of simplifying and reducing costs in the production process are always necessary.

The development of new radiation detectors using scintillation crystals, which increase response speed, dose and energy accuracy and, at the same time, the feasibility of simplifying and reducing costs in the production process are always necessary. In the CTRIPEN laboratory, pure and doped CsI crystals were grown using the Bridgman technique. The growth of a crystal is a dynamic process and involves a reciprocal exchange of atoms, or molecules, or ions. The crystal growth process, basically, consists of the transition from liquid (molten or dissolved) or gaseous state to solid state. Crystallization of a material may be divided into two steps. First, there is nucleation that consists of the formation of crystalline nuclei of critical size and, later, the growth of these critical nuclei. In our laboratory, the Bridgman technique for crystal growth is used.

\section{BRIDGMAN TECHNIQUE}

The Bridgman technique single crystal growth was developed by P. W. Bridgman, in 1925. This technique consists of fractional solidification of a material, previously melted at a temperature gradient. Bridgman technique uses the normal crystallization process and controls overcooling so that a single crystal may be obtained [1].

Normal crystallization is understood to mean the process in which a material is initially completely molten, and then progressively solidified. In this technique, the material to be grown is initially completely melted into a finely-tuned geometrical crucible, in a vertical oven, in a region of about $50{ }^{\circ} \mathrm{C}$ above its solidification point and displaced along a gradient of appropriate temperature. When the bottom of the crucible reaches the furnace region, at the melting point temperature of the material, nucleation occurs [1].

\section{SEgRegation Phenomena of IMPURITIES DURING Crystal Growing}

The intentional introduction of one or more impurities (dopants) into the host material, in the crystal growth process, may drastically modify their physical properties. The host material and the dopant are, conventionally, treated as solvent and solute, respectively, as a function of the difference in the order of magnitude of the components concentration. The solubility of these dopants is not, as a rule, equal in the different phases in the thermodynamic equilibrium, which causes the migration of these impurities from

*tmfilho@usp.br 
one phase (solid or liquid) to another (liquid or solid), during the crystallization process [1].

The process of joining with crystallization is demonstrated. Dopant migration, known as segregation, modifies the concentration of phases continuously, originating a crystalline phase concentration profile, predicted in the diagram of phases, towards the growth direction. However, since crystallization processes are not carried out with balance, other parameters associated with the process may affect the distribution of dopants, in the crystalline phase [1].

The dopants used during the research periods were: $\mathrm{Br}, \mathrm{Pb}, \mathrm{Tl}, \mathrm{Li}$. The dehumidification, growth and purification scheme of pure CsI crystals and CsI crystals plus dopants are illustrated in Fig. 1.
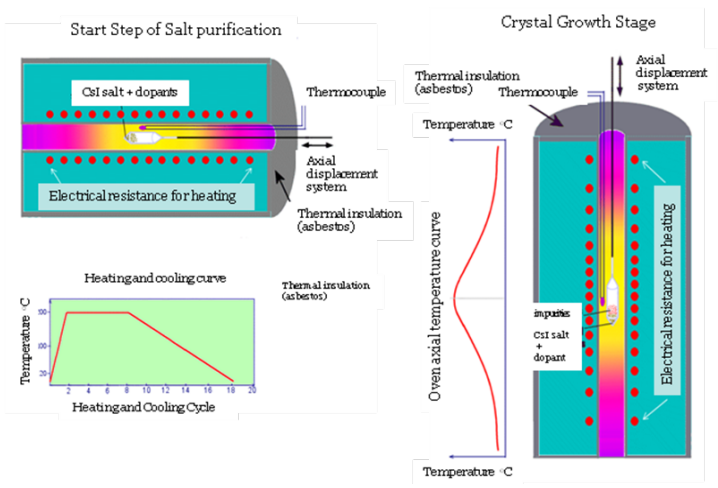

Figure 1. CsI crystals: dehumidification, growth and purification scheme [1]

\section{CSI DOPED WITH BR AND PB}
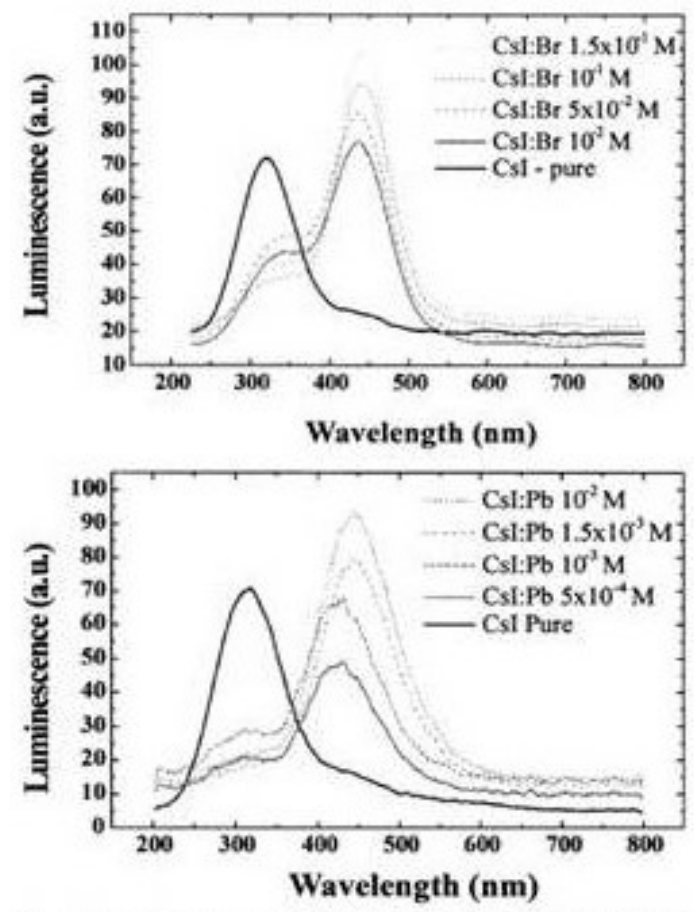

Figure 2. Luminescence emission spectra of the CsI:Br, CsI:Pb at different concentrations and pure CsI crystals 118
Transparent and uniform CsI: $\mathrm{Br}$ and $\mathrm{CsI}: \mathrm{Pb}$ crystals, $25 \mathrm{~mm}$ in diameter and $120 \mathrm{~mm}$ high were obtained at a $\mathrm{Br}$ concentration, in the range from $1.0 \times 10^{-1} \mathrm{M}$ to $5 \mathrm{X}^{-1} \mathrm{O}^{-2} \mathrm{M}$, while $\mathrm{Pb}$ concentration was $10^{-2} \mathrm{M}$ to $5 \times 10^{-4} \mathrm{M}$. Above these concentrations, the crystals were non-uniform in composition and had large opaque regions.

A predominant luminescence range near $450 \mathrm{~nm}$ and a single wide band, around $320 \mathrm{~nm}$, were found with the addition of $\mathrm{Br}$ or $\mathrm{Pb}$. These values are within the spectral sensitivity range of the photomultipliers. Thus, CsI: $\mathrm{Br}$ and CsI: Pb crystals are suitable for use as radiation detectors. Figure 2 shows the luminescence emission spectra of CsI: $\mathrm{Br}, \mathrm{CsI}: \mathrm{Pb}$ crystals, at different concentrations, and pure CsI crystal. The energy spectra and energy resolution for CsI: $\mathrm{Br}$, doped with $1 \mathrm{O}^{-1} \mathrm{M} \mathrm{Br}$, when excited with ${ }^{22} \mathrm{Na}$ and ${ }^{137} \mathrm{Cs}$, are shown in Figure 3 [2, 3, 4].
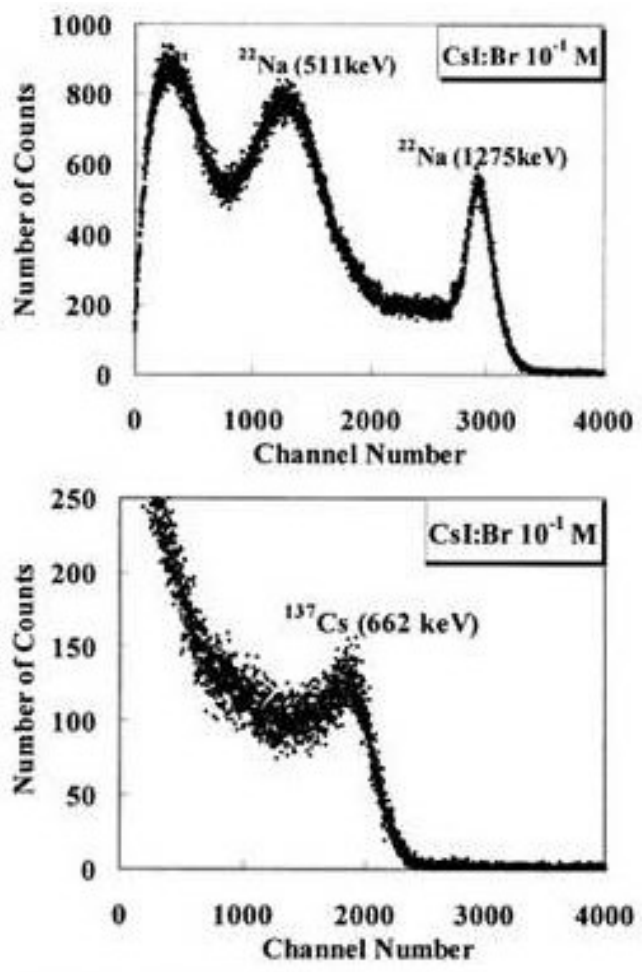

Figure 3. Energy spectra of CsI:Br $10^{-1} \mathrm{M}$

\section{CSI DOPED WITH TL}

The $10^{-3} \mathrm{M}$ molar ratio CsI (Tl) crystal was grown by the Bridgman technique at a rate of $1 \mathrm{~mm} \mathrm{~h}^{-1}$. This crystal is $20 \mathrm{~mm}$ long and $20 \mathrm{~mm}$ in diameter. Total growth time was 120 hours.

Thallium-activated cesium iodide crystal [CsI (Tl)] is used as a radiation sensor due to its favorable scintillator characteristics. It has good mechanical resistance, is poorly hygroscopic and its light emission spectrum coincides with the sensitivity region of photomultipliers and PIN-type photodiodes.

The main purpose of the CsI: Tl crystal is its use in the high energy conversion of gamma rays and X-rays into light photons capable of sensitizing photosensors. To achieve this goal, the CsI:Tl crystal is suitable 
mainly because it consists of chemical elements with high atomic masses $\left(A_{\text {cesium }}=133\right.$, A Aodine $\left.=127\right)$ and thus favoring $\mathrm{X}$-ray interactions with the multiple electronic layers photoelectric effect and Compton.

CsI (Tl) has a density of $4.51 \mathrm{~g} / \mathrm{cm} 3$ and a higher atomic number than $\mathrm{NaI}(\mathrm{Tl})$; consequently, its efficiency for gamma detection is higher. The light conversion efficiency of CsI (Tl) is, approximately, $45 \%$ of $\mathrm{NaI}(\mathrm{Tl})$ efficiency, at room temperature. It has a decay constant of, approximately, 10ns. The emission spectrum of CsI (Tl) extends from 420 to 60onm, with $540 \mathrm{~nm}$ maximum luminescence wavelength. This material is less hygroscopic than $\mathrm{NaI}$ (Tl), but it deteriorates if exposed to water or high humidity. Compared to $\mathrm{NaI}(\mathrm{Tl})$, the $\mathrm{CsI}(\mathrm{Tl})$ crystal has lower hardness and greater malleability, better resistance to severe shocks, acceleration and vibration, as well as to greater gradients or sudden changes in temperature.

In the study of the response to neutron radiation, gamma radiation, beta radiation and alpha particles (Fig. 4), the crystals were directly coupled to the photomultiplier tube (RCA MODEL 8575, 21 pins) using silicone grease (Dow Corning) with a viscosity of 0.5 McStokes, as optical interface. This ensured a uniform refractive index across the contact surface between the crystal and the photomultiplier tube. Sides of the crystal, which were not in contact with the photo-sensor, were covered with several layers of the polytetrafluoroethylene (PTFE) tape to ensure a good reflection of light. The electronic modules used for the processing the signals from the photomultiplier tube were conventional Canberra electronic.

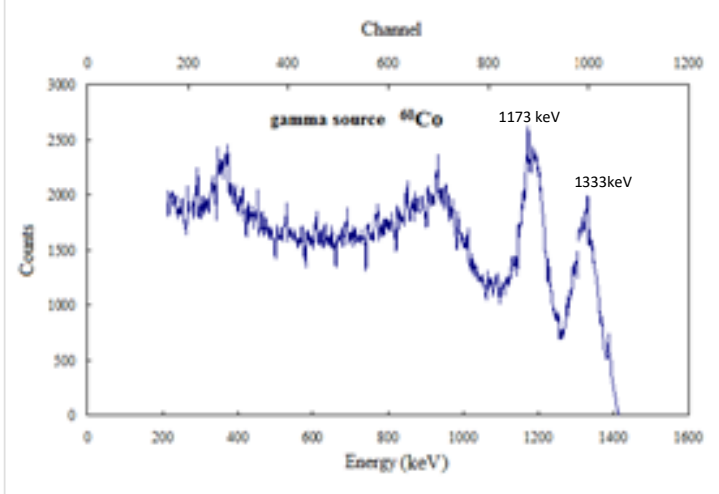

(a)

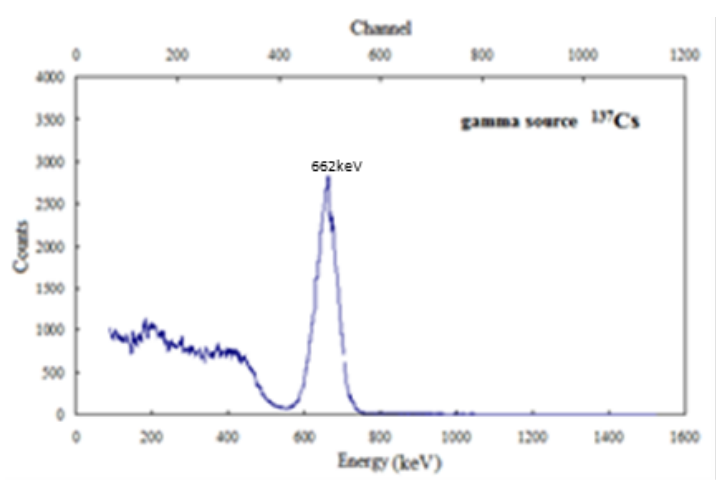

(b)

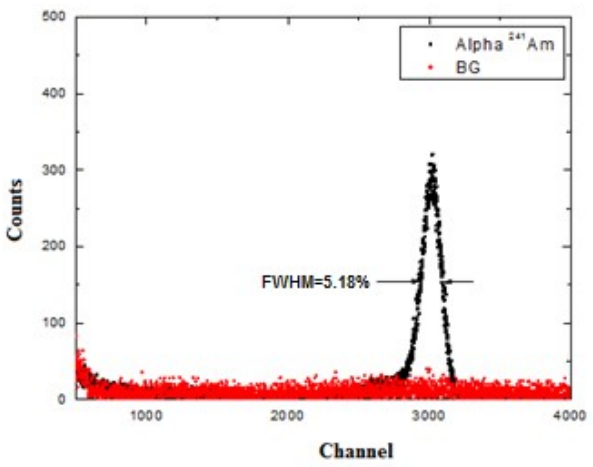

(c)

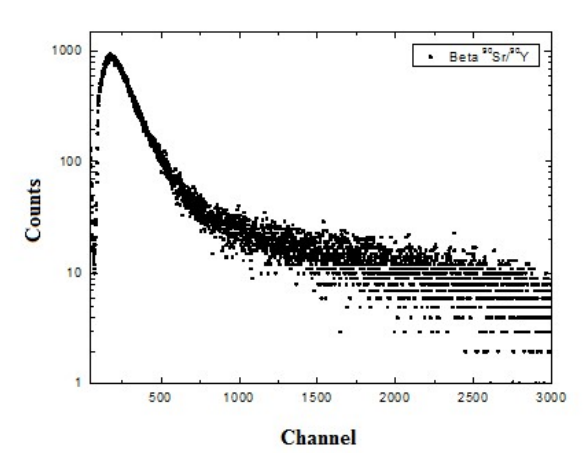

(d)

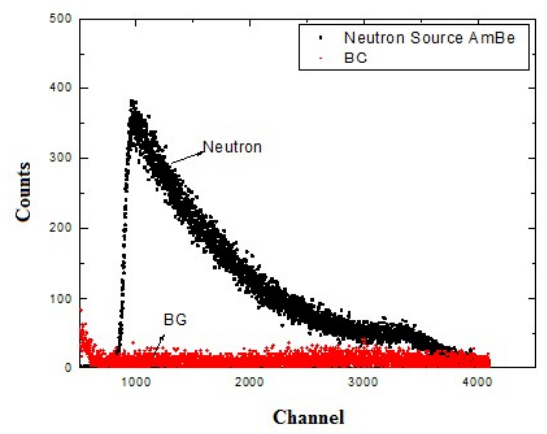

(e)

Figure 4. Energy spectra obtained for the radiation from: (a) ${ }^{60} \mathrm{Co},(b){ }^{137} \mathrm{Cs}$, gamma ray, (c) ${ }^{241} \mathrm{Am}$ alpha, (d) ${ }^{90} \mathrm{Sr}^{90} \mathrm{Y}$ beta, (e) AmBe neutron source with a CsI:Tl crystal coupled to a photomultiplier

In our laboratory, a feasibility study of these scintillators with X-ray imaging systems was performed. The CsI:Tl scintillating crystal, with a nominal concentration of $10^{-3} \mathrm{M}$, was used. The results of the X-ray diffraction analysis on the crystals were consistent with the pattern of a centered face cubic crystal (fcc) structure. Slices of $25 \times 2 \times 3 \mathrm{~mm} 3$ (length, thickness, height) of the crystal and $1 \times 2 \times 3 \mathrm{~mm} 3$, mini crystals (length, thickness, height), were utilized for the comparison in X-ray imaging systems, Fig. 5 . With these scintillating crystals, images of undesirable elements, such as metals in food packaging, were obtained. A one-dimensional array of photodiodes and the CCD (Coupled Charge Device) photosensor were 
employed. Fig. 5 presents the results obtained in the $\mathrm{Rx}$ system of images [5,6].

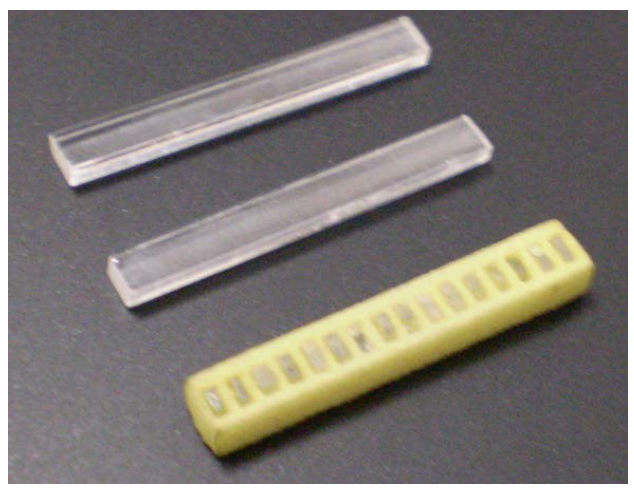

Figure 5. Slices of CsI:Tl crystal and support filled with mini crystals
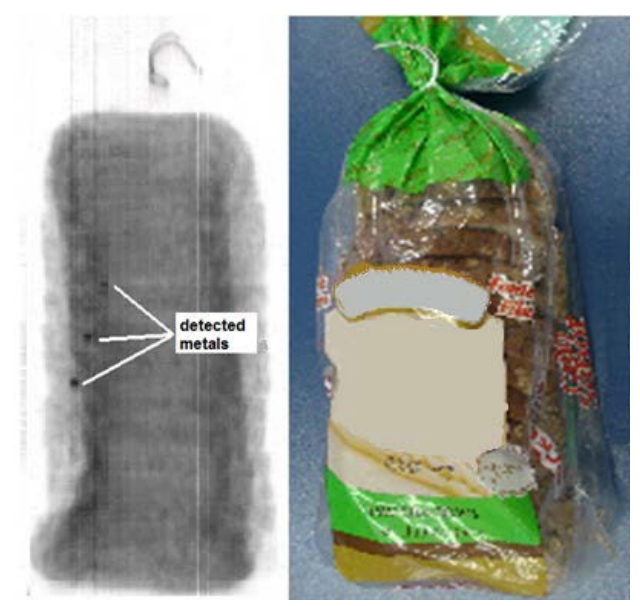

Figure 6. Image of bread package showing the result obtained by inserting a slice of $25 \times 3 \times 2 \mathrm{~mm} \mathrm{CsI}$ :Tl crystal, in the imaging system. Packing was placed under three iron objects with diameters 2, 3 and $4 \mathrm{~mm}$, seen in the left, highlighted

\section{CSI DOPED WITH LI}

Lithium-doped CsI crystals were cultured using the Bridgman vertical technique, at IPEN / CNEN-SP Institute for Nuclear and Energy Research. Lithium concentration, the doping element (Li) studied, was $10^{-3} \mathrm{M}$. These crystals were used for neutron detection tests.

Due to the lack of electric charge, the interaction of the neutron with the atomic electric field is insignificant and, therefore, the interaction of neutrons with the matter occurs, essentially, through the interaction with the nuclei.

Neutron detectors are developed based on: Elastic Scattering, Capture Reactions, Fission, Inelastic Scattering or (n, p) or (n, $\alpha)$ Reactions.

These detectors have, internally, a neutron converter to produce ionizing radiation. Converters are based on nuclear reactions, such as:

Neutron + Converter $\rightarrow$ ionizing radiation.

The converter is a material with a high cross section. The cross section is a parameter that expresses the probability of a neutron interaction with the target and it depends on the incident neutron energy [7].

Neutron response from an $\mathrm{AmBe}$ source was measured with a pure crystal, Li-doped crystal and without a crystal. The neutron source had a nominal activity of $3.7 \cdot 10^{10} \mathrm{~Bq}$ (1.0Ci of 241Am). The scintillating crystals used were cut with dimensions of $20 \mathrm{~mm}$ diameter and $20 \mathrm{~mm}$ height.

Fig. 6 shows the results of the neutron source for the photomultiplier without a crystal and for the CsI: Li crystal, using the ${ }^{60} \mathrm{Co}$ source. In Fig. 7, measurements taken using the neutron source, a pure CsI crystal and a ${ }^{6} \mathrm{Li}$ doped crystal are shown. A paraffin block to thermalize the neutrons and a sheet of $\mathrm{Cd}$, to cut the thermal neutrons, were used.

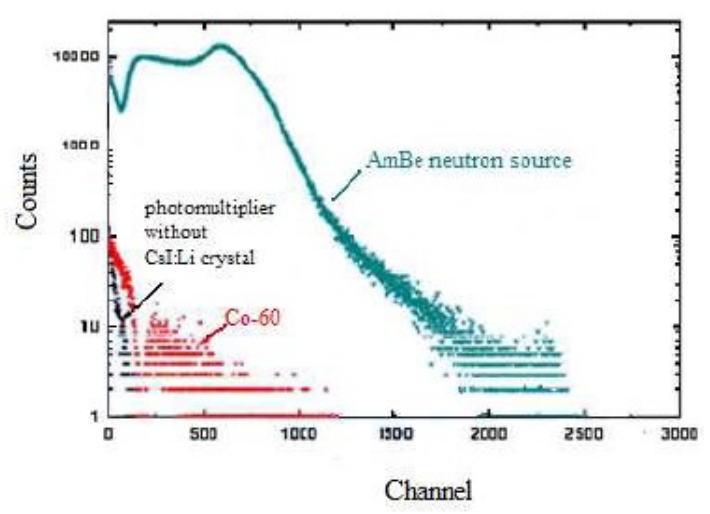

Figure 6. Pulse height spectra of the CsI:Li inorganic scintillator, from the radiation of ${ }^{6 \circ} \mathrm{Co}$ and $\mathrm{AmBe}$ sources.

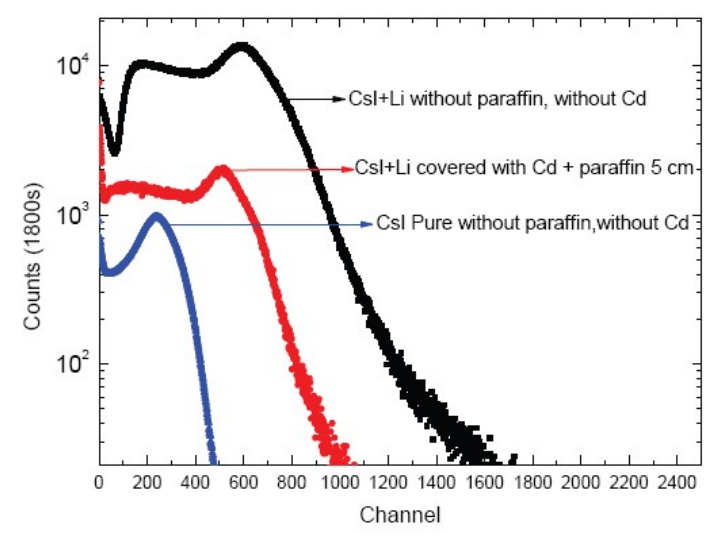

Figure 7. Results for three different measurement conditions: (a) pure CsI and a neutron source, (b) ${ }^{6} \mathrm{Li}$ doped crystal covered with paraffin and a Cd foil and a neutron source and (c) ${ }^{6} \mathrm{Li}$ doped crystal and a neutron source.

\section{CONCLUSIONS}

The vertical Bridgman technique used in this work was suitable for the growth of doped CsI crystals.

The grown crystals showed appropriate optical qualities, showing a decrease in the transmittance value as the doping element concentration increased.

The maximum emission peak luminescence spectra at $420 \mathrm{~nm}$ show a good overlap with the quantum efficiency spectrum of the two alkaline 
photomultipliers, demonstrating the feasibility of using CsI doped crystals as radiation detectors.

The addition of dopants to the CsI matrix resulted in promising crystals to be used as detectors of gamma radiation and neutron radiation. The crystals showed sensitivity in the energy range from $59 \mathrm{keV}$ to $1333 \mathrm{keV}$ and sensitivity to fast and thermal neutrons.

\section{REFERENCES}

1. M. C. C. Pereira, "Desenvolvimento de cristais baseados em iodeto de Césio para aplicação como detectores de radiação" Tese de doutorado, Universidade de São Paulo, Instituto de Pesquisas Energéticas e Nucleares, São Paulo, Brasil, 2006.

(M. C. C. Pereira, "Development of crystals based in cesium iodide for application as radiation detectors," Ph.D thesis, University of Sao Paulo, Nuclear and Energy Research Institute, Sao Paulo, Brazil, 2006.) DOI: $10.11606 /$ T.85.2006.tde-16052012-084114

2. M. C. C. Pereira, T. M. Filho, M. M. Hamada, "Development of crystals based on cesium iodide for measurements of gamma radiation and alpha particles," Nukleonika, vol. 54, no. 3, pp. 151 - 155, 2009.

Retrieved from:

http://www.nukleonika.pl/www/back/full/vol54 2009 v54n3p151f.pdf

Retrieved on: Apr. 11, 2019

3. M. C. C. Pereira, T. M. Filho, M. M. Hamada, "The effect of $\mathrm{Pb}^{2+}$ dopant in the crystal of CsI and its application as scintillation detector: A study of alpha particles,"
Radiat. Eff. Defects in Solids, vol. 167, no. 12, pp. 921 928, Nov. 2012.

DOI: $10.1080 / 10420150.2012 .723002$

4. M. C. C. Pereira, T. M. Filho, "Scintillation Characteristics of CsI Crystal Doped Br under Gamma and Alpha Particles Excitation," Mater. Sci. Appl., vol. 5, no. 6, pp. 368 - 377, May 2014. DOI: $10.4236 / \mathrm{msa} .2014 .56042$

5. M. C. C. Pereira, T. M. Filho, V. M. Lopes, J. R. Berretta, J.P. N. Cárdenas, "Scintillation Response of CsI:Tl Crystal Under Neutron, Gamma, Alpha Particles and Beta Excitations," in Proc. 2015 Int. Nuc. Atl. Conf. (INAC 2015), Sao Paulo, Brazil, 2015.

Retrieved from:

https://inis.iaea.org/collection/NCLCollectionStore/_P ublic/47/032/47032097.pdf? $\mathrm{r}=1 \& \mathrm{r}=1$

Retrieved on: Mar. 15, 2019

6. M. C. C. Pereira, T. M. Filho, J. R. Berretta, C. H. Mesquita, "Characteristics of the CsI:Tl Scintillator Crystal for X-Ray Imaging Applications," Mater. Sci. Appl., vol. 9, no. 2, pp. 268 - 280, Feb. 2018.

DOI: $10.4236 / \mathrm{msa} .2018 .92018$

7. T. M. Filho, M. C. C. Pereira, J. R. Berretta, J. P. N. Cárdenas, "Study of a Li doped CsI scintillator crystal as a neutron detector," J. Phys. Conf. Ser. vol. 630 , no. 1, 2015 DOI: $10.1088 / 1742-6596 / 630 / 1 / 012010$

8. M. C. C. Pereira, T. M. Filho, J. P. N. Cárdenas, "Inorganic scintillation crystals for neutron detection," in Proc. 2013 3rd Int. Conf. Adv. Nucl. Instrum., Measurement Methods and their Appl. (ANIMMA), Marseille, France, 2013.

DOI: 10.1109/ANIMMA.2013.6727878 